\title{
Development and Validation of High Performance Unshrouded Cetrifugal Impeller
}

\author{
Wei-Chung Chen, M.Williams, John K. Paris, G.H.Prueger \\ (The Boeing Company, Rocketdyne Propulsion and Power)
Canoga Park, CA 91309-7922 \\ R.Williams (NASA/MSFC) \\ Huntsville, AL.
}

\begin{abstract}
The feasibility of using a two stage unshrouded impeller turbopump, Figure 1, to replace the current three-stage RLV engine shrouded impeller hydrogen pump has been evaluated from the standpoint of turbopump weight reduction and overall payload improvement. These advantages are a byproduct of the higher tip speeds that an unshrouded impeller can sustain. The issues associated with the effect of unshrouded impeller tip clearance on pump efficienc and head have been evaluated with onedimensional tools and full three-dimensional CFD analysis. Unshrouded impeller rotordynamic fluid reaction forces and coefficients have been established through time dependent CFD simulation of the whole 360 degree impeller with different rotor eccentricities and whirling ratios. Unlike the shrouded impeller, the unshrouded impeller forces are evaluated as the sum of the pressure forces on the blade and the pressure forces on the hub using the CFD results. The turbopump axial thrust control has been optimized by adjusting the 1st stage impeller backend wear ring seal diameter and diverting the 2nd stage backend balance piston flow to the proper location. The structural integrity associated with the high tip speed has been checked $b$ analyzing a 3D-Finite Element Model at maximum design conditions (6\% higher than the design speed). This impeller was fabricated and tested in the NASA/MSFC
\end{abstract}

water-test rig. The experimental data will be compared with the analytical predictions and presented in another paper ${ }^{7}$. The experimental data provides validation data for the numerical design and analysis methodology. The validated numerical methodology can be used to help design different unshrouded impeller configurations.

\section{Introduction}

Rocket engine weight reduction is constantly being pursued in order to place more payload into orbit at lower cost. Turbopump weight is typically $25 \%$ to $30 \%$ of the gross engine weight and thus is a good candidate for weight reductions. Turbopump weight can be broken down into rotor assembly, $20 \%$, and housing assembly $80 \%$. The key item to note is that, although, the housing makes up the greatest portion of the turbopump weight it is driven by the turbopump rotor element envelope, i.e. diameter and axial length, Figure 1. Reduction in rotor element diameter can be done through an increase in tip speed capability. This can be accomplished through an increase in material capability or removal of the impeller shroud to reduce blade stresses. NASA Monograph SP-8109 indicates that for a hydrogen pump using forged titanium impellers an increase in tip speed from $2000 \mathrm{feet} / \mathrm{sec}$ to $2500 \mathrm{feet} / \mathrm{sec}$ is achievable with removal of the shroud. This relates to a $25 \%$ reduction in diameter to 
achieve the same impeller stage pressure rise.

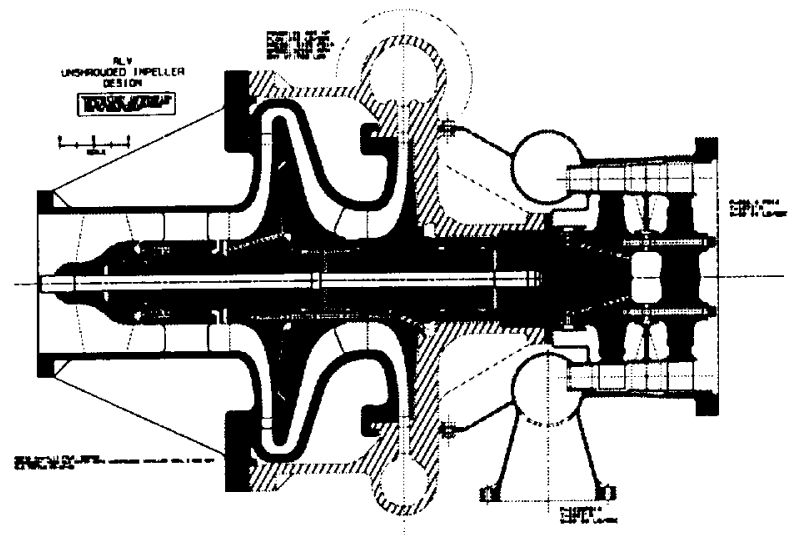

Figure 1: Unshrouded Impeller Turbopump Layout

Reduction in rotor assembly axial length is best achieved through elimination of a stage or rotor element. This can be accomplished by increasing the head achieved by one rotor element through an increase in the head coefficient or through increased tip speed. The use of high head unshrouded impeller technology has the potential to significantl reduce turbopump weight by allowing the use of higher tip speeds and decrease of a rotor stage.

\section{Blade Design to Minimize Clearance impacts}

A number of papers have been published on the impact of tip clearance on unshrouded (semi-open) impellers for compressors and pumps. In 1972 Rocketdyne completed an evaluation of shrouded and unshrouded impeller performance using the J2 liquid oxygen turbopump. Water tests were completed with a shrouded impeller and an impeller with the shroud removed at various tip clearances. The performance impact of a $10 \%$ increase in the tip clearance resulted in a $12 \%$ decrease in efficiency $y^{\prime}$. Y. Senoo ${ }^{2}$ wrote in 1987 that a tip clearance change from 0 (shrouded) to $10 \%$ of the impeller exit width decreases efficiency by $4 \%$ for compressors. The different impact of tip clearance on these turbopumps indicates that impeller design parameters can impact the efficiency defect. In 1997 Johannes Lauer, et. al. ${ }^{3}$ describes an experimental study of 14 semi-open impellers of different design. The results were not conclusive, but indicated that the blade number, and exit angle had the largest impacts on tip clearance sensitivity.

\section{Rotordynamic Coefficient Prediction}

Stable turbomachinery operation depends on the damping of the rotor motion ${ }^{4}$. Currently, rotordynamic stability parameters are estimated by using bulk flow theories and small perturbation (quasi-steady) assumptions. A well-established experience base with unshrouded impeller rotordynamics does not exist.

To help understand the unshrouded impeller's rotordynamic performance, Enigma's computational rotordynamic methodology $y^{5,6}$ was applied to the unshrouded impeller. This method directly simulates the rotor whirling motion (no quasi-steady assumptions) and can be, in principle, applied to large eccentricity whirl problems.

For Navier-Stokes based rotordynamic calculations, the impeller shaft/hub moves with an imposed whirling harmonic motion, Figure 2, and the flow equations are integrated time-accurately until reaction force time periodicity is observed. The fluid reaction force vector time history is calculated; the force history can then be post-processed and decomposed into normal and tangential components. Because of the direct simulation of the moving hub, the flow model must consist of the complete three-dimensional geometry (full 360 degrees in circumference). 


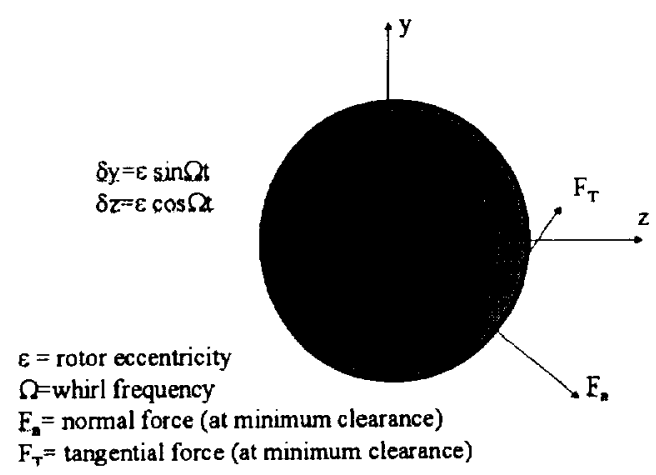

Figure 2: Whirling impeller rotor

Historically, impeller coefficients have rarely been a design driver from a rotordynamics stand point in liquid hydrogen turbomachinery, for two primary reasons. First, the magnitude of the impeller coefficients is proportional to fluid density, and are therefore typically predicted to be quite low relative to the other coupling elements (in fact, sometimes ignored) in liquid hydrogen. And second, Rocketdyne has usually incorporated shrouded impellers into their turbomachinery designs, which intuitively would be expected to have lower rotordynamic coefficients than comparably sized unshrouded impellers. The validity of the second assumption above can be assessed by examining the normal and tangential force data predicted by the CFD analysis. By applying a second degree polynomial curve fit to the data in Figure 3, rotordynamic coefficients for the subject unshrouded impeller can be extracted.

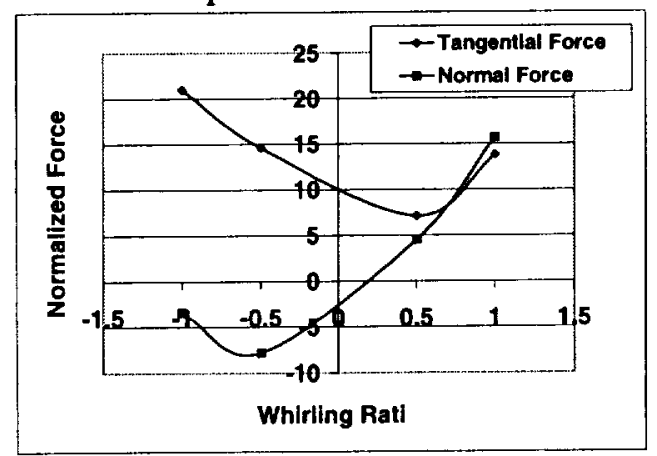

Figure 3: Computed hub+blades normal and tangential forces
By comparing these coefficients to those predicted for the SSME HPFTP impellers, which are comparable in size to the RLV turbopump impellers, a quantitative assessment can be made regarding how significant these impeller forces may be for an unshrouded impeller. Table 1 lists this comparison.

Table 1: Rotordynamic coefficient comparison, unshrouded/shrouded impellers

\begin{tabular}{|l|c|c|}
\hline & $\begin{array}{c}\text { RLV } \\
\text { unshrouded }\end{array}$ & $\begin{array}{c}\text { SSME HPFTP } \\
\text { shrouded }\end{array}$ \\
\hline Kxx & $-50,330 \mathrm{lb} /$ in & $-20,144 \mathrm{lb} /$ in \\
\hline Kxy & $95,877 \mathrm{lb} /$ in & $8,774 \mathrm{lb} /$ in \\
\hline Cxx & $15 \mathrm{lb}-$ sec/in & $6 \mathrm{lb}$-sec/in \\
\hline
\end{tabular}

These data, Table 1, show that the unshrouded impeller direct stiffness $\left(\mathrm{K}_{\mathrm{xx}}\right.$ ) and direct damping coefficients $\left(\mathrm{C}_{\mathbf{x x}}\right)$ are about 2.5 times larger than the same coefficients currently used to simulate the SSME HPFTP rotordynamic performance. However the cross-coupled stiffness coefficient $\left(\mathrm{K}_{\mathrm{xy}}\right)$, which affects turbopump stability, for the unshrouded impeller is almost 11 times the same coefficient for the SSME HPFTP. Not only is this predicted coefficient now so large such that it cannot be ignored, but it is, in fact, over twice as large as the cross-coupled stiffness attributed to the turbine Alford force, which must always be considered in a rotordynamic stability assessment. While the increased damping coefficient would partially offset the destabilizing effect of this large crosscoupling term, it's net effect would certainly be much more destabilizing than the comparably combined effects for a shrouded impeller (stability representing a balance of whirl drivers, Kxy, and whirl dampers, Cxx).

Another point of qualification needs to be made regarding this comparison. The 
current best estimate impeller coefficients for the SSME turbopumps are calculated by scaling empirical data derived from rig testing at $\mathrm{Cal} \mathrm{Tech}$. Analytically predicted impeller coefficients, from either bulk flow or CFD models, have historically not compared well with empirical data, and in fact have typically tended to significantl under-predict the measured coefficients. Therefore, it is possible (perhaps even likely) that the difference between the rotordynamic coefficients of shrouded and unshrouded impellers is even larger than that suggested herein, if empirical data were available for both.

In summary, it appears very likely that all the rotordynamic coefficients associated with unshrouded impellers could be significantly larger than those of comparable shrouded impellers. Furthermore, and consistent with what would be intuitively expected, the potential destabilizing effects in particular could be much more significant. This makes it imperative that impeller coefficients be accurately accounted for in the rotordynamic model of any turbopump utilizing unshrouded impellers, and probabl $\gamma$ makes the pump stability issue, which can be problematic for high performance hydrogen pumps in any case, that much more challenging.

With regard to the current numerical procedure for unshrouded impellers, more validation is needed to completely assess the impact on the presented layout.

\section{Pump weight study}

\section{Weight Calculation Assumptions}

The following equations were used to define the turbopump weight based on stage number considerations and diameter:

$$
\begin{aligned}
& D_{\text {Crossover }}=\left\{\begin{array}{lll}
1.27 \times D_{\text {Im peller }} & 1 & \text { stage } \\
1.45 \times D_{\text {Im peller }} & 2 & \text { stage } \\
1.65 \times D_{\text {Im peller }} & 3 & \text { stage }
\end{array}\right. \\
& L_{P_{\text {ump }}}=\left\{\begin{array}{l}
32 \times\left(\frac{D_{\text {Crossover }}}{24}\right){ }^{1} \text { stage } \\
40 \times\left(\frac{D_{\text {Crossover }}}{24}\right) \\
2 \text { stage } \\
48 \times\left(\frac{D_{\text {Crossover }}}{24}\right)
\end{array}\right. \\
& \text { Weight }_{\text {pump }}=2100 \times\left(\left(^{D_{\text {crossore }} / 24}\right)^{1.5} \times\left(L_{\text {Pump }} / 40\right)^{1.25}\right.
\end{aligned}
$$

The crossover or diffuser diameter, which sets the housing major dimension, is dependent on the impeller diameter through an increase of the diffusion requirement for a given head coefficient. The scaling parameters of $1.27,1.45$, and 1.65 are based on the fact that the more stage pump the impeller discharge is less tangential and more radial flow therefore require bigger diffuser to diffuse the flow. The pump length is set based on the number of stages where the scaling parameters of 32,40 , and 48 are based on the RS68 fuel turbopump. Weight should then vary with the diameter and length to give a cubic relation to volume. Rocketdyne experience has determined that the relationship is closer to a 2.75 power law and this was used.

Figure 4 shows the result of the weight trade completed to define the impeller tip speed and staging. The RLV 3 stage baseline is shown at approximately 2500 pounds using the prescribed weight assumptions and 32,000 RPM. Prior to review of the operating speed to take into account turbine constraints, a design point was selected at 42,000 RPM and an impeller tip speed of $2600 \mathrm{feet} / \mathrm{sec}$. This would have shown a pump weight improvement of 1086 pounds. 
Due to constraints in turbine materials the speed was limited to 32,000 RPM. With a fixed pump speed and a fixed discharge pressure increasing tip speed increases impeller diameter and results in a heavier turbopump even with a reduction in one pump stage. Taking into account the weight correlation and turbine constraint on RPM resulted in a 2200 feet/sec tip speed to minimize weight and arrive at an impeller design which had a reasonable head coefficient ( $\Psi$ ) and eye-to-tip ratio for operating flow range considerations. This is the 2-stage baseline design point. This design shows a 626 pound decrease in turbopump weight from the RLV 3 stage baseline design and a 147 pound decrease from a 3 stage RLV design with equivalent head coefficient.

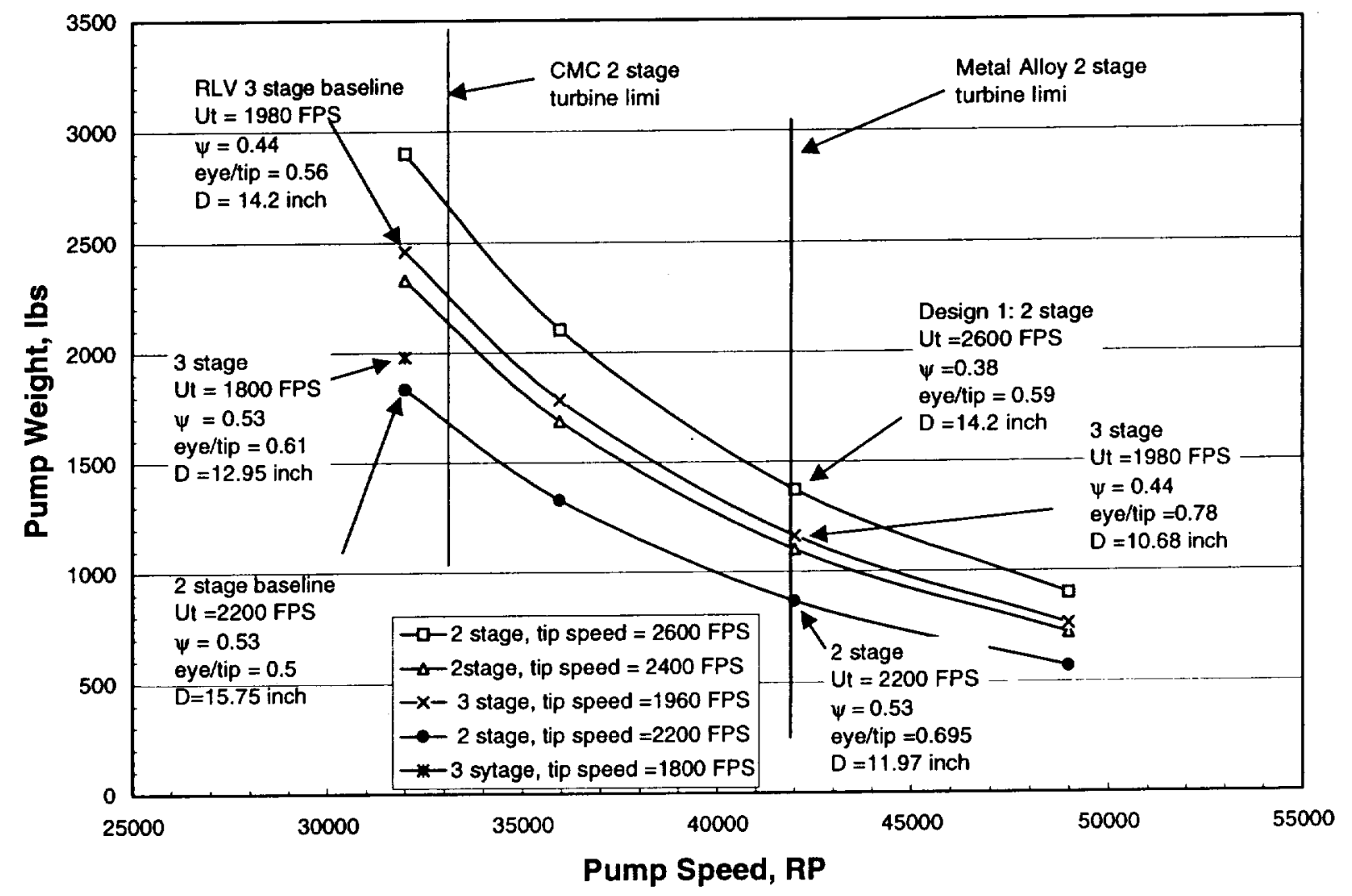

Figure 4: Weight trade study for design point selection

\section{Axial thrust calculation}

A concern with unshrouded impellers is the balance of axial thrust. Shrouded impellers have similar $\mathrm{k}$-factors in the front and rear shrouds, whereas an unshrouded impeller does not. This imbalance has direct impact on the position of wear rings and the balance piston design. Figure 5 shows the rotor force distribution. The first stage impeller is balanced with a wear ring located at a 14.2 inch diameter. The total unbalanced force that a balance piston must counteract is 614,606 pounds. An evaluation was made as to the routing of the balance piston sump to achieve this requirement, Figure 7 . The initial evaluation was to determine if rotor balance could be achieved with routing the balance piston secondary flow across the second stage impeller alone. This is represented in the curve with the square symbols. As can be seen, the balance piston 
would be operating in a closed position with the high pressure orifice and there is no force margin available to account for load

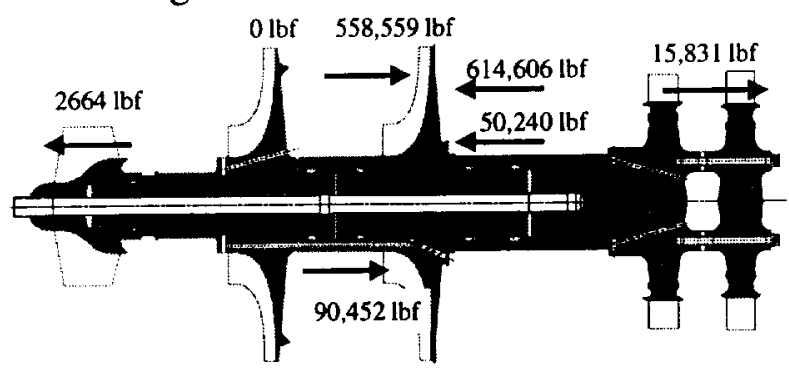

Figure 5: Rotor Force Balance

uncertainties. Two other cases were then run with routing of the balance piston sump to the inlet of the first stage impeller. This adds some complexity to the mechanical arrangement but as can be seen in Figure 6 dramatically increases the balance piston capability. The circle symbol and triangle symbol curves both represent return to the inlet of the first stage. The triangle symbol curves shows larger balance piston capability through the use of antivortex ribs in the balance piston cavity. This effectivel reduces the swirl in this cavity and decreases the pressure drop increasing the net force.

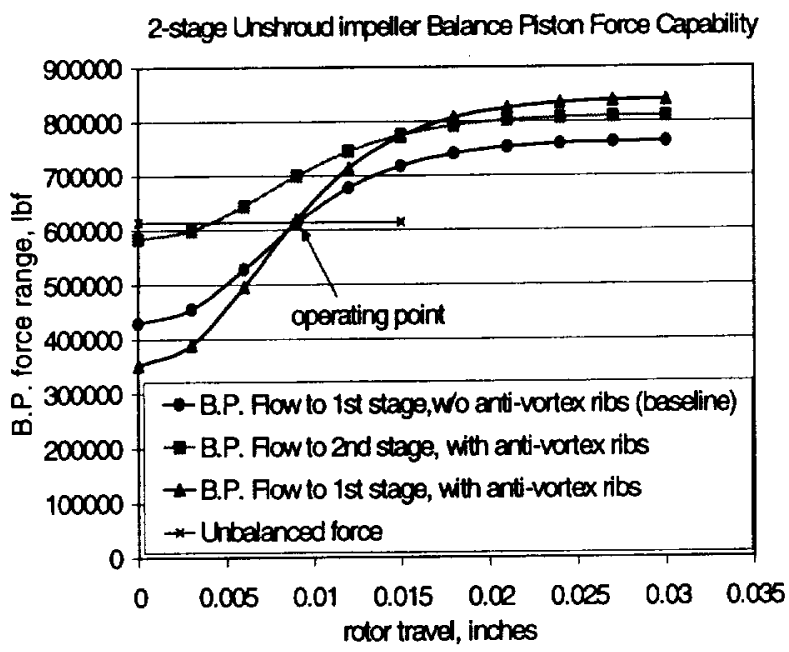

Figure 6: Balance piston configuration study

The negative consequence of the antivortex ribs is an increase in balance piston secondary flow. Figure 7 shows this impact.
The use of antivortex ribs increases has a net result of increasing the balance piston flow rate by $20 \%$ over the configuration without antivortex ribs. This increase in flow rate would result in a $1.23 \%$ drop in pump efficiency. At this point in the design it was felt that the decrease in performance was not worth the increase in balance piston capability. As the design matures this decision would have to be revisited. It should be noted that the baseline configuration with balance piston sump return to the inlet of the first stage without antivortex ribs has only a $0.8 \%$ negative effect on pump efficiency. This balance piston shows adequate-operating range with the unbalanced force at approximately $50 \%$ of the force range. This sort of mechanical arrangement was successfully shown in the Rocketdyne MK-49 hydrogen pump for an orbital transfer vehicle system.

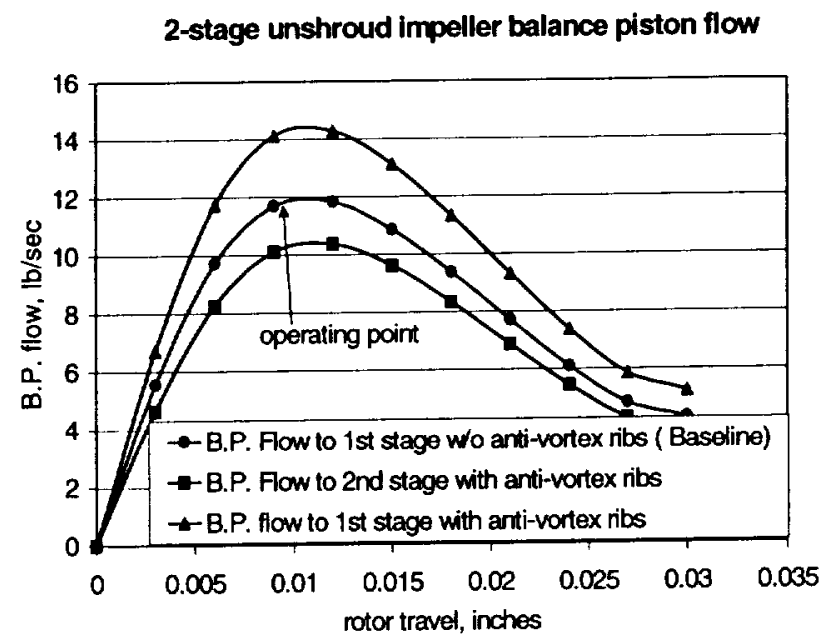

Figure 7: Balance piston flow rate

\section{Structural Analysis}

A structural assessment was performed on the unshrouded impeller to demonstrate that the structural factors of safety and calculated fatigue life meet the required structural criteria (factor of safety). Hub bust analysis was done using two-dimensional axisymmetric finite element models (FEM) following the Rocketdyne burst 
methodology. Three hub thickness values ranging from 0.200 to 0.400 inch were evaluated; the results of which are presented in Table 2.

Table 2: Burst Analysis Results

\begin{tabular}{|c|c|c|c|}
\hline & \multicolumn{3}{|c|}{ Hub Thickness } \\
\hline & $\mathbf{0 . 2 0 0}$ in. & $\mathbf{0 . 3 0 0}$ in. & $\mathbf{0 . 4 0 0}$ in. \\
\hline FS $_{\text {burst }}$ & 2.02 & 1.90 & 1.77 \\
\hline $\mathbf{N}_{\text {allow }}$ (rpm & 41,385 & 39,490 & 38,187 \\
\hline $\begin{array}{c}\mathbf{V}_{\text {tip allow }} \\
\text { (ft/sec) }\end{array}$ & 2,996 & 2,859 & 2,764 \\
\hline
\end{tabular}

Three-dimensional FEMs were used to determine the yield, ultimate, and high cycle fatigue $(\mathrm{HCF})$ factors of safety. Results are shown below in Table 3.

Table 3: Blade Factors of Safety

\begin{tabular}{|c|c|c|c|c|}
\hline & \multicolumn{3}{|c|}{ Hub Thickness (inches) } & \multirow{2}{*}{$\begin{array}{c}\text { Required } \\
\text { Safety } \\
\text { Factor }\end{array}$} \\
\hline & 0.200 & 0.300 & 0.400 & \\
\hline Yield & 1.92 & 1.95 & 1.89 & 1.10 \\
\hline Ultimate & 1.79 & 1.82 & 1.77 & 1.4 \\
\hline HCF & 2.40 & 3.26 & 3.33 & 1.4 \\
\hline
\end{tabular}

The initial design employed a hub thickness of 0.200 inch. High stresses were obtained at the blade tip with pressure loading on the blades and hub. Hub bending caused the high stresses, which also existed for the 0.300 inch hub. The stresses obtained using the 0.400 inch hub were satisfactory. Optimization of the hub for weight or other considerations would require a more detailed analysis.

The items evaluated met the required structural criteria. It was determined that the blade thickness could be reduced by $5 \%$ and still maintain adequate margins on the factors of safety. The maximum allowable tip speed, taking hub burst and blade life into account was determined to be approximately $2700 \mathrm{ft} / \mathrm{s}$.

\section{Conclusions}

The test data for this particular impeller was not available yet at this paper publication, however based on the existing data and some CFD predictions the following conclusions can be made:

(1) Unshrouded impellers can be used to reduce the pump overall weight and increase the overall payload.

(2) CFD predicts that unshrouded impellers create unfavorable rotordynamic coefficients in the pump. How this impacts on the pump rotordynamic stability needs more study.

(3) Unshrouded impeller axial thrust balance is similar to that of a shrouded impeller; need to identify the proper location for the balance piston sump.

(4) Structural analysis usually favors the unshrouded impeller because the common high stresses located near the L.E. regions of a shrouded impeller no longer exist.

(5) Impeller efficiency is lower for unshrouded impellers. The efficiency loss can be minimized by reducing the shroud clearance through pump development program.

\section{References}

1 R.K. Hoshide and C.E. Nielson, "Study of Blade Clearance Effects on Centrifugal Pumps," Contract \#: NAS3-13311, Report \#: NASA CR-120815, NASALewis Research Center

${ }^{2} \mathrm{Y}$. Senoo and M. Ishida, "Deterioration of Compressor Performance Due to Tip Clearance of Centrifugal Impellers," Journal of Turbomachinery, January 1987, Vol. 109, pp. 55-61

3 Johannes Lauer, et. al., "Tip Clearance Sensitivit of Centrifugal Pumps with Semi-Open Impeller," 1997 ASME Fluids Engineering Division Summer Meeting, FEDSM97-3366. 
4 Williams, M., "A Helmholtz Pressure Equation Method for the Calculation of Unstead Incompressible Viscous Flows", Int. J. Numer. Meth. Fluids, Vol. 14, 1992, pp.1-12.

5 M.Williams, W.Chen, L. Brozowski and A. Eastland, "Three-Dimensional Finite Difference Method for Rotordynamic Fluid Forces on Seals," AIAA J., vol. 35, No. 8, pp. 1417-1420, 1997.

${ }^{6}$ Hiwata Akira, Tsujimoto Yoshinobu , "Theoretical Analysis of Fluid Forces on an Open-Type Centrifugal Impeller in Whirling Motion" ForthInternational Symposium on Pumping Machinery, May 29, 2001 New Orleans, LA.

7 R. Williams, "Comparison of Unshrouded Impeller Analysis and Experiment" $37^{\text {th }}$ AIAA/ASME/SAE/ASEE Joint Propulsion Conference, Salt Lake City, Utah. 8-11 July, 2001. 